\title{
The Influence of Topography on the Stability of Shelfbreak Fronts
}

\author{
M. SUSAN LOZIER \\ Division of Earth and Ocean Sciences, Duke University, Durham, North Carolina \\ MARK S. C. REED \\ North Carolina Supercomputing Center, Research Triangle Park, North Carolina
}

(Manuscript received 26 August 2003, in final form 31 August 2004)

\begin{abstract}
In an attempt to understand the degree to which the stability of a shelfbreak front, characterized by continuous horizontal and vertical shear, is affected by topography, a linear stability analysis was conducted for a range of frontal jets and bottom-slope configurations. Three-dimensional perturbations superposed on a continuously stratified shelfbreak front were investigated using linearized, hydrostatic primitive equations. For all model runs in the study, the frontal instability mode, which is the fastest-growing mode for a baroclinic flow, was not influenced by the bottom: Retrograde, prograde, and flat-bottom jets all share the same stability characteristics. In contrast, weakly baroclinic jets are strongly influenced by bottom topography. The presence of a bottom slope stabilizes prograde jets and destabilizes retrograde jets, a difference attributed to the orientation of the isopycnals relative to the bottom slope. Temporal and/or downstream changes in the bottom slope and/or background stratification are shown to produce sizeable changes in the instability of a weakly baroclinic jet.
\end{abstract}

\section{Introduction}

Shelfbreak currents are generally characterized by sharp property fronts that delineate coastal from open ocean waters. Significant temporal variability and spatial variability of these fronts lead to the mixing and exchange of coastal and open ocean waters. As such, an understanding of the source of this variability would improve our estimates of onshore/offshore exchange and our ability to predict frontal evolution, a prediction desirable for a host of applications, including those associated with navigation, fisheries, weather forecasting, and national defense. A much-studied shelfbreak current that exemplifies this highly variable nature is situated in the Middle Atlantic Bight (Beardsley et al. 1985). Over 20 years ago it was suggested that frontal instabilities were responsible for the observed variability of this front (Flagg and Beardsley 1978); however, until recently, instability models were unable to capture modes with appreciable growth rates (Flagg and Beardsley 1978; Gawarkiewicz 1991) that matched observational growth rates (Garvine et al. 1988). Studying

Corresponding author address: M. Susan Lozier, Nicholas School of the Environment and Earth Sciences, Division of Earth and Ocean Sciences, Box 90230, Duke University, Durham, NC 20078-0227.

E-mail:mslozier@duke.edu the stability of a continuously stratified shelfbreak frontal current governed by linearized primitive equations, Lozier et al. (2002; hereinafter referred to as LRG) found unstable modes with growth rates on the order of 1 day over a wide range of idealized background conditions applicable to the Middle Atlantic Bight. Lending credence to the supposition that instabilities are a source of the variability associated with the Middle Atlantic Bight shelfbreak current are two recent observational studies. From a study of ADCP data collected over an 18-month period in the shelfbreak region south of New England, Fratantoni and Pickart (2003) concluded that the most probable cause of the observed mesoscale variability was baroclinic instability of the shelfbreak jet. Local wind forcing and tidal rectification, as well as the offshore effects of Gulf Stream rings, topographic Rossby waves, and Gulf Stream meandering, were all discounted as possible sources for the observed variability. Furthermore, from an analysis of surface drifters that were entrained upstream of the Middle Atlantic Bight, Lozier and Gawarkiewicz (2001) found ubiquitous meandering and cross-frontal exchange that were restricted by neither locale nor season and which existed even in the absence of Gulf Stream rings-all characteristics consistent with frontal instability.

Though the LRG study focused specifically on the Middle Atlantic Bight, with model topography based on the shelfbreak bathymetry just south of Nantucket 
Shoals, a wide range of background conditions for the velocity and density fields were examined. A parameter study was conducted by varying the strength, width, and depth of the background velocity field and the extent of density stratification, a range of conditions believed to be sufficient to extend the applicability of the results to other shelfbreak frontal currents. However, shelfbreak currents are characterized not only by differences in background conditions of the velocity and density field, but also by their underlying topography. Shelfbreak currents vary according to the steepness of the continental slope, as well as by their flow direction relative to the slope. Prograde currents flow such that their cyclonic flank is in shallow (onshore) water, while retrograde jets flow with their cyclonic flank in the deeper (offshore) water [Mooers et al. 1978; see Li and McClimans (2000) for an alternate definition]. Past studies have indicated that both of these factors, slope steepness and flow direction relative to the bathymetric slope, can influence flow instability (e.g., Orlanski 1969; Blumsack and Gierasch 1972; Li and McClimans 2000). Thus, the extent to which the LRG results are generally applicable to other shelfbreak frontal currents is dictated by the effect of topography on the stability of those currents. Past studies on the influence of topography on oceanic flows have generally been restricted to either barotropic or baroclinic flows and conducted with approximated dynamics. The overall goal of this study is to assess the influence of topography on the stability of a shelfbreak frontal current characterized by continuous horizontal and vertical shear and governed by primitive equation dynamics.

To characterize the frontal instabilities, a linearized primitive equation stability model is employed to determine the three-dimensional propagation of perturbations superposed on a unidirectional mean flow field, which varies continuously across stream and with depth. The role of topography in stabilizing or destabilizing the flow is investigated for both retrograde and prograde jets. In addition, the effect of stratification on such stability is investigated. The relevant background for this study is given in the next section. Methods are outlined in section 3 , followed by a discussion of results in section 4 and a summary in section 5 .

\section{Background}

In a seminal study almost 40 years ago, Pedlosky (1964) derived a necessary condition for the instability of continuously stratified, quasigeostrophic flows characterized by both horizontal and vertical shear. Following the formulation used in Pedlosky (1979), we consider a background zonal flow, defined by a geostrophic streamfunction $\Psi(y, z)$ superposed with a perturbation streamfunction given by $\varphi(x, y, z, t)$, so that the evolution of the flow field is given by $\psi(x, y, z, t)=\Psi(y, z)$ $+\varphi(x, y, z, t)$. In the absence of friction and for smallamplitude perturbations,

$$
\frac{\partial \bar{E}}{\partial t}=-\frac{\partial E(\phi)}{\partial t}=\int_{0}^{z_{0}} \int_{-1}^{1} \rho_{0}\left(\overline{u v} \frac{\partial U}{\partial y}+\overline{v \theta} \frac{\partial \Theta}{\partial y}\right) d y d z,
$$

where $E(\phi)$ and $\bar{E}$ represent the total perturbation energy and the total mean energy, respectively. The mean and perturbation potential temperatures are given by $\Theta$ and $\theta$, respectively, and $u(v)$ represents the zonal (meridional) perturbation velocity. The mean zonal velocity is denoted by $U, \rho_{0}$ is the background density, and the top and bottom boundaries are at $z=z_{0}$ and $z=0$, respectively. All quantities are expressed in nondimensional form. Conversion of mean to perturbation energy (and vice versa) depends upon perturbation momentum and heat fluxes acting upon the background velocity and potential temperature fields. For perturbation energy to increase with time, the perturbation or eddy fluxes must be downgradient such that there is a decrease in the mean gradient fields. The perturbation fluxes can be expressed in terms of the background field and the meridional displacement of fluid elements, $\eta(x, y, z, t)$. In particular, the perturbation heat flux at the bottom boundary can be expressed as

$$
\overline{v \theta}=\left(\frac{\partial U}{\partial z}-S \frac{\partial \overline{\eta_{B}}}{\partial y}\right) \frac{\partial \overline{\eta^{2}} / 2}{\partial t} \text { at } z=0,
$$

where $\eta_{B}$ is the nondimensional height of the bathymetry as measured from $z=0\left(\eta_{B}>0\right)$ and $S$ is the Burger number. For an unstable field, where the meridional displacements increase with time, this formulation shows that the sign of the perturbation heat flux depends upon the difference between the background velocity shear and the bottom slope. Expressing all the perturbation fluxes as a function of $\eta$ and imposing the constraint that in the absence of friction the $x$-averaged zonal momentum is preserved, a necessary condition for instability can be derived (Pedlosky 1979):

$$
\begin{aligned}
0= & \int_{0}^{z_{0}} \int_{-1}^{1} \rho_{0} \frac{\partial \Pi_{o}}{\partial y}\left(\frac{\partial}{\partial t} \overline{\eta^{2}}\right) d y d z \\
& +\int_{-1}^{1}\left(\frac{\rho_{0}}{S} \frac{\partial U}{\partial z} \frac{\partial}{\partial t} \overline{\eta^{2}}\right)_{z=z_{0}} d y \\
& -\int_{-1}^{1}\left[\rho_{0}\left(S^{-1} \frac{\partial U}{\partial z}-\frac{\partial \eta_{B}}{\partial y}\right) \frac{\partial}{\partial t} \overline{\eta^{2}}\right]_{z=0} d y
\end{aligned}
$$

where $\Pi_{0}$ is the background potential vorticity. As noted by Pedlosky $(1964,1979)$, the vanishing of this integral is a necessary condition for instability. Such a condition can be met in a number of ways, depending upon the background flow and the flow configuration (viz., the vertical shear) at the top and bottom boundaries. Our interest is focused on the bottom boundary where it is apparent that the bottom slope plays a significant role in this integral constraint. This role can be understood in a geometric context when the thermal 
wind relationship is used to rewrite the innermost term (in parentheses) of the final term in Eq. (2.3) as

$$
\left(S^{-1} \frac{\partial U}{\partial z}-\frac{\partial \eta_{B}}{\partial y}\right)=\frac{L}{\varepsilon D}\left[\left(\frac{\partial z_{*}}{\partial y_{*}}\right)_{\Theta_{*}}-\frac{\partial h_{B}}{\partial y_{*}}\right] \text { at } z=0,
$$

where $L(D)$ is the horizontal (vertical) length scale, $\varepsilon$ is the Rossby number, $h_{B}$ is the dimensional bathymetry (as measured from $z=0),\left(\partial z_{*} / \partial y_{*}\right)_{\Theta_{*}}$ is the slope of the isotherms in the basic state, and the terms on the righthand side are all dimensional, as denoted by the asterisk. The stability of the flow can now be seen to depend on the bottom slope relative to the slope of the isotherms. The link between this geometry and the stability process is made by combining Eqs. (2.2) and (2.4) so that the perturbation heat flux at the bottom boundary can be expressed as

$$
\overline{v \theta}=C\left[\left(\frac{\partial z_{*}}{\partial y_{*}}\right)_{\Theta_{*}}-\frac{\partial h_{B}}{\partial y_{*}}\right] \frac{\partial \overline{\eta^{2}}}{\partial t} \text { at } z=0,
$$

where $C$ is a positive constant. Thus, whether the perturbation flux is downgradient (contributing to the growth of the perturbation energy) or upgradient (contributing to the growth of the energy of the mean flow) depends directly upon the geometry of the isotherms relative to the bottom boundary. This relationship will be made more explicit in the following sections.

The incorporation of topography in early studies of flow instability necessarily used approximated dynamics and approximated shear; that is, the flow fields were purely barotropic, purely baroclinic, or layered in the vertical direction. Studies on the topographic influence on shelfbreak frontal instability also fell into these categories. Using a two-layer model and the geostrophic momentum approximation (Hoskins 1975), Flagg and Beardsley (1978) found a rapid decrease in growth rates with increasing bottom slopes for the baroclinic instability of a retrograde jet, a decrease so large as to make the growth rates unrealistic. Using the same approximated dynamics but with a more sophisticated layered model, Gawarkiewicz (1991) also found that topography stabilized a retrograde jet. In their study of a purely barotropic slope current, Li and McClimans (2000) found that even mild slopes stabilized both retrograde and prograde jets. Recent advances in numerical methods (Moore and Peltier 1987) have allowed for the solution of instability equations using primitive equation dynamics and continuous shear in both the horizontal and vertical flow fields (Barth 1994; Xue and Mellor 1993; Samelson 1993). With such a stability model, Xue and Mellor (1993) studied the Gulf Stream flow in the South Atlantic Bight and found that topography stabilized the mixed baroclinic/barotropic instability of this prograde jet. Barth (1994) used a similar model to study the stability of coastal upwelling jets and found that topography had little influence on a frontal instability mode captured by the primitive equation dynamics (Moore and Peltier 1989), whereas topography generally stabilized the traditional baroclinic mode (at low wavenumbers) of a prograde jet. Our work follows from these studies in that we employ a jet characterized by continuous horizontal and vertical shear and governed by primitive equation dynamics, yet our focus is exclusively on the influence of topography on the stability of a shelfbreak frontal current for which jet orientation, slope strength and stratification are varied.

\section{Methods}

Our computation of the linear instabilities of a background geostrophic jet with continuous stratification is based on the method developed by Moore and Peltier (1987) and modified by Xue and Mellor (1993) to include a topographic slope. In this section we will briefly describe the model equations and solution methods. The reader is referred to Moore and Peltier (1987), Xue and Mellor (1993), and LRG for details on this method and computation.

\section{a. Model equations and solution method}

The model used in this study consists of a steady background current flowing along the slope of idealized bathymetry (Fig. 1). The model uses a Cartesian coordinate system, where $x$ is the offshore axis (positive offshore), $y$ is the alongshore axis (positive upstream), and $z$ is the vertical axis (positive upward). Note that this coordinate system differs from that presented in the last section, in which the system used by Pedlosky (1964, 1979) was preserved. For this formulation of the shelfbreak jet, our preference is to orient the $y$ axis in the alongshore direction. Here, water depth is given by $h(x)$ and the alongshore background flow is given by $V(x, z)$. The background flow is in thermal wind balance with a mean density field $\rho(x, z)$ according to $f_{0} V_{z}=B_{x}$, where $f_{0}$ is a constant Coriolis parameter, $B$ is the mean buoyancy defined by $B=-g \rho(x, z) / \rho_{0}$, with $\rho_{0}$ being the reference density and $g$ the gravitational acceleration. To assess the stability of this basic state, three-dimensional velocity and density perturbations are superposed onto a two-dimensional background velocity and density field. The evolution of the perturbations is governed by the hydrostatic primitive equations, linearized about a geostrophic background state:

$$
\begin{aligned}
u_{t}+V u_{y}-f_{0} v & =-\pi_{x}, \\
v_{t}+u V_{x}+V v_{y}+w V_{z}+f_{0} u & =-\pi_{y}, \\
0 & =-b+\pi_{z}, \\
u_{x}+v_{y}+w_{z} & =0, \quad \text { and } \\
b_{t}+u B_{x}+V b_{y}+w B_{z} & =0 .
\end{aligned}
$$




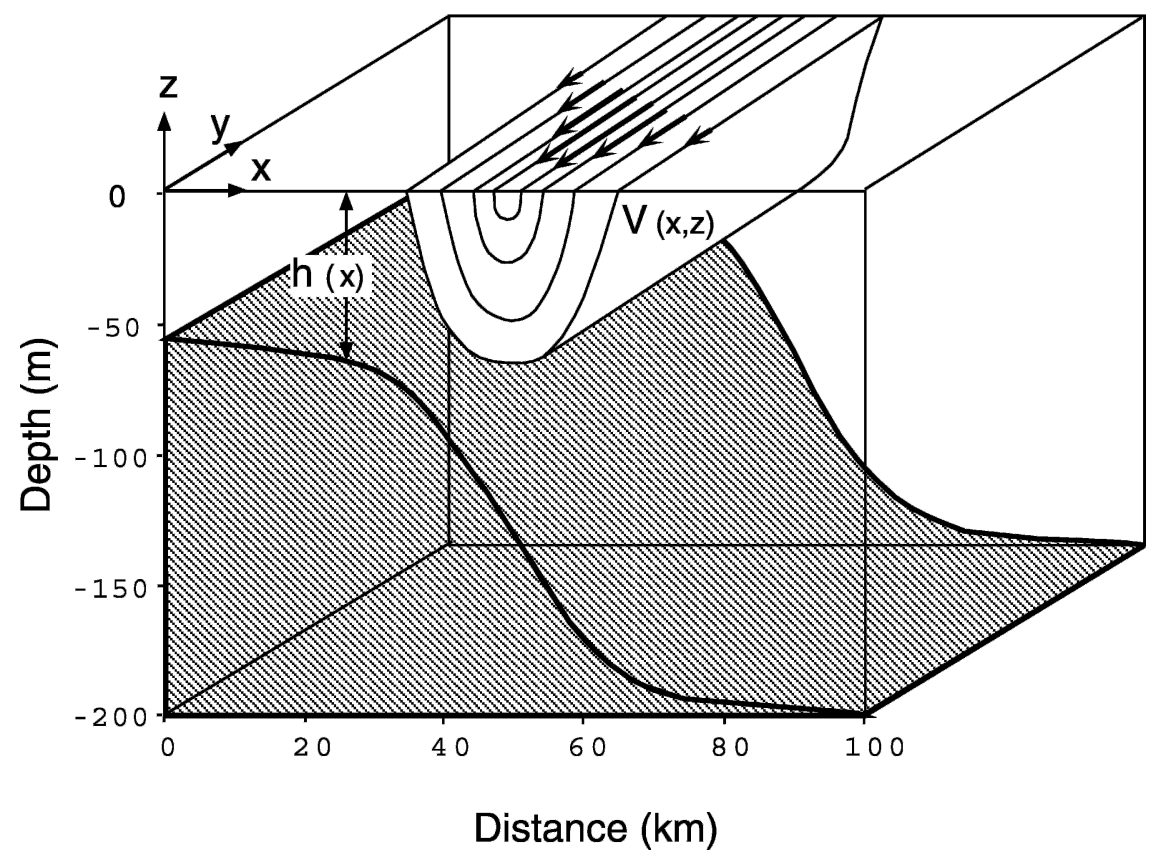

FIG. 1. Schematic of retrograde model jet and bathymetry used in this study. Definitions of $V(x, z)$ and $h(x)$ are given in section 3b. All model jets are centered at $x=50 \mathrm{~km}$.

The Cartesian components of the perturbation velocity are $(u, v, w) ; \pi$ is perturbation pressure divided by $\rho_{0}$, and $b$ is the perturbation buoyancy. Equations (3.1)(3.3) are the equations of motion with no frictional or external forces, and Eqs. (3.4) and (3.5), derived from the conservation of mass, represent the continuity equation for an incompressible fluid and the conservation of density, respectively. Boundary conditions stipulate that normal flow across solid boundaries is zero $\left[u=0\right.$ at $x=0$ and $w=-u h_{x}$ at $\left.z=-h(x)\right]$ and that disturbances vanish at distances far from the coast. In addition, the rigid-lid approximation $(w=0$ at $z=0)$ is imposed. [Note: For our model, we set $z=0$ at the sea surface, which differs from the formulation used in section 2 in which $z=0$ defines the bottom boundary. We chose to follow the Pedlosky (1979) formulation in section 2.]

To transform the irregular grid created by the bathymetry into a regular rectangular grid (in the $x$ and $z$ directions), a mapping is applied to Eqs. (3.1)-(3.5). Such a mapping facilitates the use of basis functions, which are described below. The mapping, applied according to $\zeta=1+z / h(x)$, yields a new vertical velocity $\omega$ given by $\omega=w-(\zeta-1) u h_{x}$ (Xue and Mellor 1993). With this mapping it is convenient to define transport variables: $\left(u^{*}, v^{*}, b^{*}\right)=(u h, v h, b h)$. In addition, all variables are cast in dimensionless form using the following scaling: $x^{\prime}=x / L_{0}, y^{\prime}=y / L_{0}, \zeta^{\prime}=\zeta, t^{\prime}=t f_{0}, u^{\prime}=$ $u^{*} / u_{0}, v^{\prime}=v^{*} / u_{0}, \omega^{\prime}=\left(L_{0} / u_{0}\right) \omega, h^{\prime}=h / H_{0}, \pi^{\prime}=\pi H_{0} /$ $\left(u_{0} f_{0} L_{0}\right), b^{\prime}=b^{*} H_{0} /\left(u_{0} f_{0} L_{0}\right), B^{\prime}=B /\left(H_{0} N_{0}^{2}\right)$, and $V^{\prime}=$ $V / V_{0}$, where $L_{0}$ and $H_{0}$ are the horizontal and vertical length scales, respectively; $u_{0}$ is the typical perturbation velocity times $H_{0} ; N_{0}$ is the Brunt-Väisälä frequency; and $V_{0}$ is the maximum of the background velocity $V(x, z)$. For all model runs $L_{0}=100 \mathrm{~km}, H_{0}=200 \mathrm{~m}$, and $f_{0}=9.37 \times 10^{-5} \mathrm{~s}^{-1}$ (corresponding to a latitude of $\left.40^{\circ} \mathrm{N}\right)$. Solutions for the three-dimensional perturbations are sought in the form:

$$
\begin{aligned}
\left(u^{\prime}, \omega^{\prime}\right) & =\operatorname{Re}\left\{\left[u^{*}(x, \zeta), \omega^{*}(x, \zeta)\right] e^{i(\sigma t+l y)}\right\} \quad \text { and } \\
\left(v^{\prime}, \pi^{\prime}, b^{\prime}\right) & =\operatorname{Re}\left\{i\left[v^{*}(x, \zeta), \pi^{*}(x, \zeta), b^{*}(x, \zeta)\right] e^{i(\sigma t+l y)}\right\},
\end{aligned}
$$

where $\sigma$ is the complex frequency $\left(\sigma=\sigma_{r}+i \sigma_{i}\right), l$ is the alongfront wavenumber, the starred variables are the structure functions in $x$ and $z$, and $\operatorname{Re}\{\}$ denotes the real part of the expression inside the curly braces. Substitution of Eqs. (3.6) and (3.7) into Eqs. (3.1)-(3.5) yields

$$
\left(\sigma+R_{0} l V\right) u^{*}-v^{*}=-h \pi_{x}^{*}+(\zeta-1) h_{x} b^{*},
$$

$$
\begin{aligned}
\left(\sigma+R_{0} l V\right) v^{*}-u^{*}(1- & \left.R_{0} V_{x}\right) \\
- & R_{0} V_{\zeta} \omega^{*}=-l h \pi^{*}, \\
-b^{*}+\pi_{\zeta}^{*} & =0, \\
u_{x}^{*}-l v^{*}+\omega_{\zeta}^{*} & =0, \quad \text { and } \\
\left(\sigma+R_{0} l V\right) b^{*}-S B_{x} u^{*}-S B_{\zeta} \omega^{*} & =0,
\end{aligned}
$$

where $R_{0}=V_{0} /\left(f_{0} L_{0}\right)$ is the Rossby number for the background flow and $S$ is the Burger number, defined as $\left[N_{0} H_{0} /\left(f_{0} L_{0}\right)\right]^{2}$. For the numerical calculation of Eqs. (3.8)-(3.12), the background flow and the perturbations 
are confined to a domain bounded by $x=0, x=100$ $\mathrm{km}$, the sea surface $(\zeta=1)$, and the seafloor $(\zeta=0)$ (Fig. 1). The size of the domain was chosen to balance the need for computational affordability with the need for all perturbations to be vanishingly small at the channel walls. In addition, to provide for no normal flow, $u^{*}$ is set to 0 at $x=100 \mathrm{~km}$. With these boundary conditions, Eqs. (3.8)-(3.12) form an eigenvalue problem with a complex frequency given by $\sigma$ and eigenmodes given by Eqs. (3.6) and (3.7). The solution of this eigenvalue problem is achieved with a combination of Galerkin and Fourier collocation schemes. To obtain a solution, structure functions [e.g., $u^{*}(x, z)$ ] are spectrally decomposed into orthogonal sets of trigonometric basis functions in the vertical $(\zeta)$ and cross-shelf $(x)$ directions, as detailed in Xue and Mellor (1993). For numerical solution these expansions are truncated at a finite number of spectral modes $M$. These expansions are substituted into Eqs. (3.8)-(3.12) and manipulated to produce a standard matrix eigenvalue equation, which is solved by standard linear algebra methods for a specified background field, truncation level, and wavenumber. All model runs for this study used $M=44$. Readers are referred to LRG for a discussion of model convergence. Model solutions yield growth rate $\left(-\sigma_{i}\right)$, phase speed $\left(-\sigma_{r} / l\right)$, and modal structure of the instabilities at each wavenumber.

\section{b. Jet velocity profiles and bathymetry}

The background velocity field, modeled as a crossshelf Gaussian waveform that exponentially decays with depth, is expressed as

$$
V(x, z)=V_{0} \exp \left[-\sigma_{x}\left(x-x_{0}\right)^{2}-\sigma_{z}\left(z-z_{0}\right)\right],
$$

where $\sigma_{x}\left[=4 \ln \left(V_{0} / 0.1 \mathrm{~m} \mathrm{~s}^{-1}\right) / x_{d}^{2}\right]$ and $\sigma_{z}\left[=\ln \left(V_{0} / 0.1\right.\right.$ $\left.\left.\mathrm{m} \mathrm{s}^{-1}\right) / z_{d}\right]$ are formulated such that the $10 \mathrm{~cm} \mathrm{~s}^{-1}$ isotach will fall at distances $x_{d} / 2$ from $x_{0}$ and $z_{d}$ from $z_{0}$. For all model runs, the center of the jet is placed at $x=$ $50 \mathrm{~km}\left(x_{0}=50 \mathrm{~km}\right)$ and the maximum jet velocity is at the surface $\left(z_{0}=0 \mathrm{~m}\right)$. The width and depth of the 10 $\mathrm{cm} \mathrm{s}^{-1}$ isotach are specified for each model run by the variables $x_{d}$ and $z_{d}$, respectively. The background density field $B(x, z)$ is set by the velocity field and the specification of density at the offshore boundary and the condition of thermal wind balance. At the offshore boundary, the stratification is assumed to be linear $\left(B_{z}\right.$ is constant) for all model runs, with the deepest isopycnal set at $\rho=1.0275 \mathrm{~g} \mathrm{~cm}^{-3}$ (Linder 1996). To create changes in the background stratification, the uppermost isopycnal $\rho_{\text {shallow }}$ is varied at the eastern boundary. For brevity, values of $\rho_{\text {shallow }}$ will be expressed using sigma- $t$ units.

Using these parameters, two qualitatively different types of jets were produced: an idealized Middle Atlantic Bight frontal jet with strong vertical shear and one with very weak vertical shear, such that it could be characterized as a weakly baroclinic jet. The former was studied extensively in LRG, where it was shown that the modal structures associated with this jet are often surface-trapped, except for the very lowest of wave numbers. To probe the interaction of unstable modes and the bottom slope, the latter jet was also chosen for this study. The same functional form, as given above, was used for both jets. They differ only in their value of $\sigma_{z}$ [Eq. (3.13)], which controls the strength of the vertical shear.

The bathymetry used in our model study was determined from the fit of a hyperbolic tangent functional form (Xue and Mellor 1993) to the bathymetric measurements in the Nantucket Shoals region (Linder 1996). This fit is given by

$$
h(x)=H_{s}+0.5\left(H_{d}-H_{s}\right)\left\{1+\tanh \left[\left(x-x_{m}\right) / \alpha\right]\right\},
$$

where $H_{s}$ (shelf depth) $=60 \mathrm{~m}, H_{d}$ (maximum domain depth $)=200 \mathrm{~m}, x_{m}$ (location of maximum slope $)=50$ $\mathrm{km}$, and $\alpha$ (lateral extent of slope) $=15 \mathrm{~km}$. The offshore asymptotic depth in the Middle Atlantic Bight is on the order of $2000 \mathrm{~m}$, but we have used $200 \mathrm{~m}$ in our model study to reduce the computational grid. A sensitivity study to offshore depth found negligible differences between model runs using $200 \mathrm{~m}$ as the offshore depth and those using depths in excess of $200 \mathrm{~m}$. To study model sensitivity to bottom slope and to isolate quantities that are significant along the seafloor, the bathymetry of the model was modified by varying $\alpha$ in Eq. (3.14) above. The range of bathymetric slopes, produced by varying $\alpha$ from $5 \mathrm{~km}$ (steep) to a value of 25 $\mathrm{km}$ (less steep), is shown in Fig. 2. It is important to note that for our study, as for LRG, we have chosen to keep the frontal jet's centerline position fixed, to maintain constant stratification at the offshore boundary and to maintain symmetrical horizontal velocity shear. Each of these constraints is made for the purpose of simplification and could be relaxed in future studies of this frontal system.

\section{c. Prograde and retrograde jets}

To understand how flow direction relative to the bottom slope affects flow stability, the velocity and density fields of the retrograde jets were constructed to be mirror images to the prograde fields, with the only exception being the orientation of the isopycnals to the bottom slope. Given the structure of the density field imposed by the thermal wind balance, isopycnals associated with a retrograde surface-intensified jet slope in the opposite direction from the bathymetric slope (Fig. 2) while the isopycnals associated with a surface-intensified prograde jet slope in the same direction as the bathymetric slope. To create equivalent interior fields, the velocity field was simply reversed while the following procedure was used for the density fields. The density field for the retrograde jet was computed by speci- 

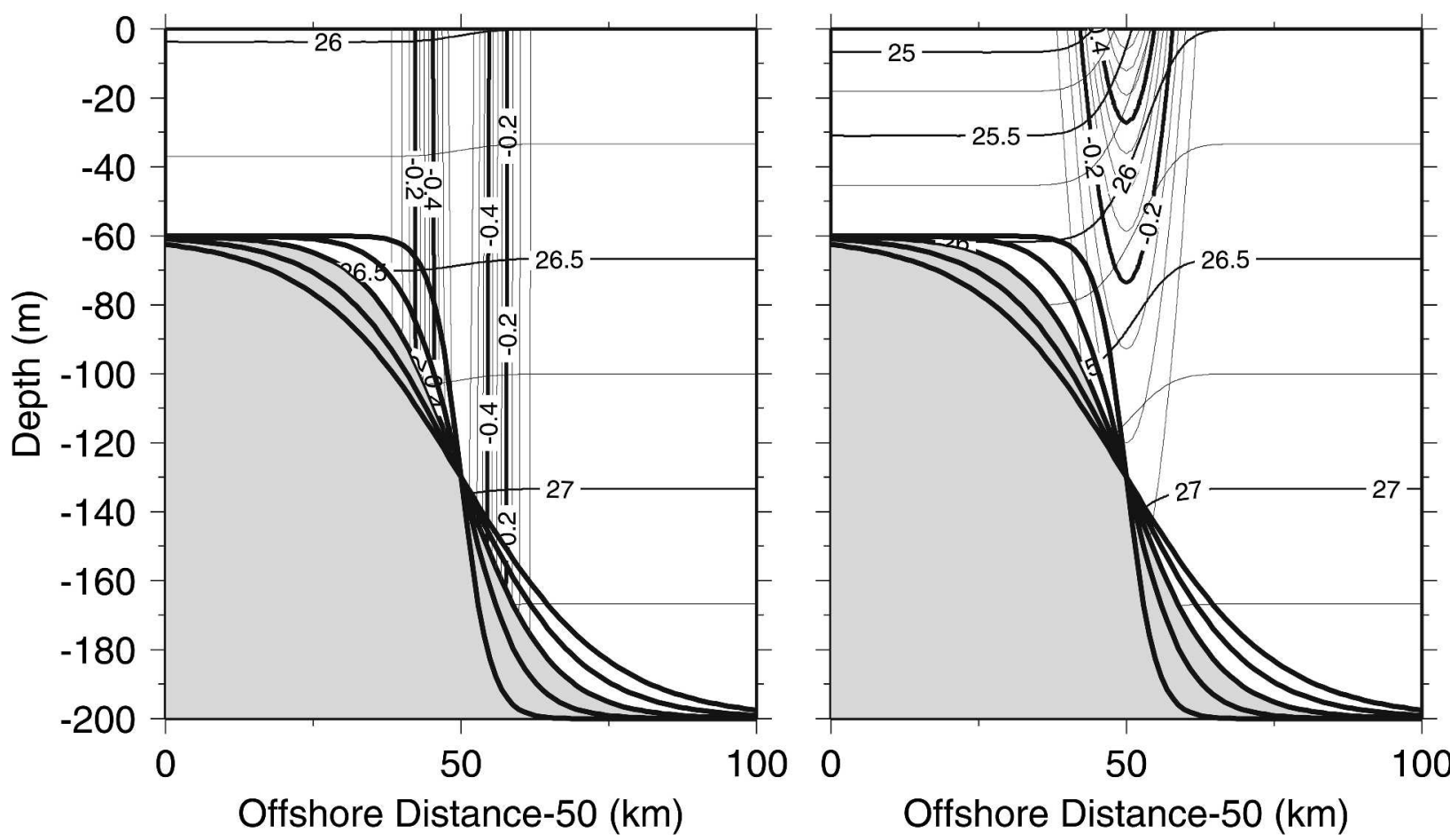

FIG. 2. Velocity and isopycnals typical of (left) a weakly baroclinic jet and (right) a strongly baroclinic jet for the standard bathymetry (shaded gray). Also shown (with black lines) are the bathymetry profiles that result by varying the slope parameter $\alpha$ [see Eq. (3.14)] from $5 \mathrm{~km}$ (steepest) to $25 \mathrm{~km}$ (least steep) in increments of $5 \mathrm{~km}$. Note how the slope of the isopycnals is opposed to the slope of the underlying bathymetry for this retrograde jet. Annotated velocity contours are at $0.2 \mathrm{~m} \mathrm{~s}^{-1}$. Isopycnals are denoted by their sigma- $t$ units.

fying the boundary conditions along the eastern border and then integrating westward using the thermal wind relation. The density values obtained along the western boundary for the retrograde jet were then specified as the eastern boundary conditions for the prograde jet. A westward integration from these boundary conditions yielded a "mirror image" of the retrograde density field.

\section{Results}

In this section we first present the model results for the weakly baroclinic jet, illustrating the effect of topography on both retrograde and prograde jets marked by either weak or strong stratification. Second, the results of the strongly baroclinic jet are presented. As mentioned above, in all cases the retrograde and prograde jets were constructed with equivalent potential vorticity fields in the interior. Thus, the necessary condition for instability [Eq. (2.3)] will be met equally by the retrograde jet and its prograde counterpart in terms of interior fields and the upper boundary condition. Thus, differences between the two jets will be attributed to differences in the orientation of their isopycnals relative to the bathymetric slope. We will specifically study how this difference affects the growth of the per- turbation field. Our focus will be on the relationship expressed in Eq. (2.5), which for our study (using isopycnal surfaces rather than isentropic surfaces and an alongshore jet rather than a purely zonal jet) is rewritten as

$$
\overline{u \rho}=-C\left[\left(\frac{\partial z}{\partial x}\right)_{\rho}-\frac{\partial h_{B}}{\partial x}\right] \frac{\partial \overline{\eta^{2}}}{\partial t}
$$

where $C$ is a positive constant, $\partial h_{B} / \partial x<0$ for our model configuration $\left(h_{B}=H_{d}-h\right.$; see Fig. 4, described later) and $(\partial z / \partial x)_{\rho}$ denotes the slope of the background isopycnals. For the following discussion, it is useful to note that the background density field for a retrograde (prograde) jet has $\partial \bar{\rho} / \partial x>0(\partial \bar{\rho} / \partial x<0)$, as illustrated in Fig. 2 for a retrograde jet, yielding positive (negative) isopycnal slopes $(\partial z / \partial x)_{\rho}$ for the retrograde (prograde) jet.

As has been demonstrated from energy analyses in past studies (Xue and Mellor 1993; Barth 1994), it is expected that the unstable perturbations in this study will derive their energy from both the vertical and horizontal shear. We can use the ratio of the internal Rossby radius of deformation $r_{i}$ to the horizontal length scale of the flow field $L_{h}$ as a measure of the importance of available potential energy relative to kinetic energy as the energy source for the perturbation growth (LRG). For all runs in this study with the weakly baro- 


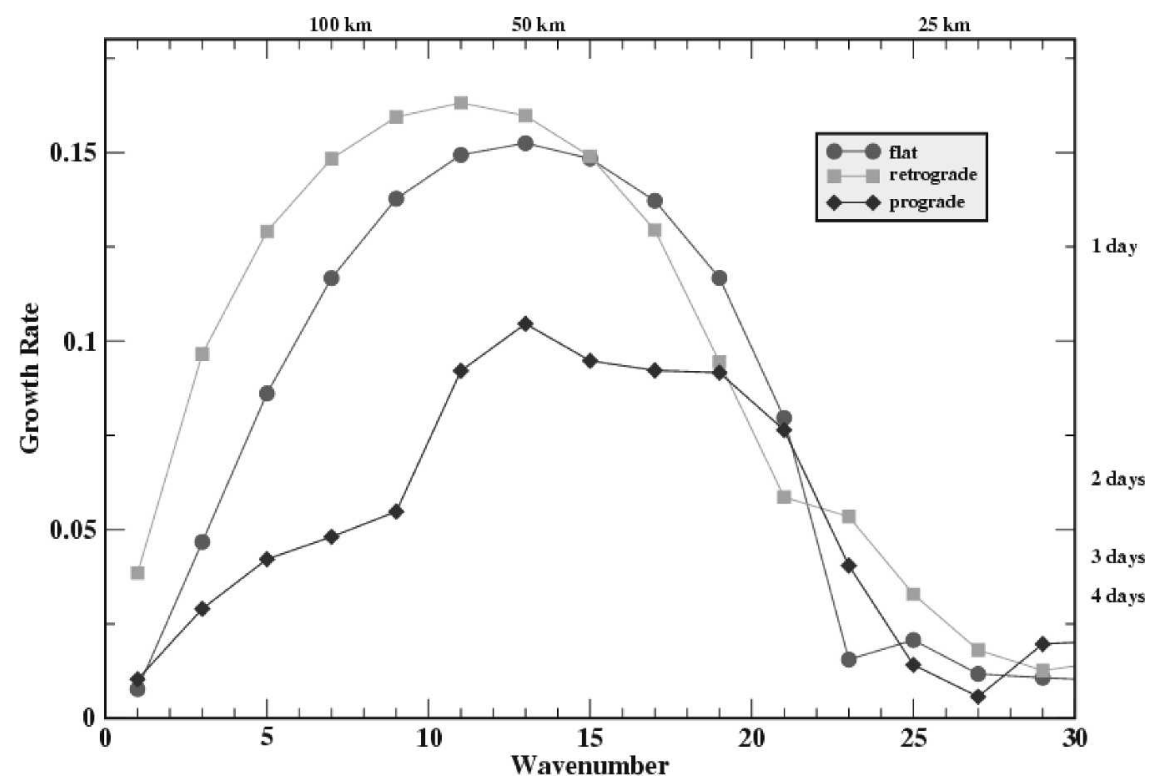

FIG. 3. Plot of growth rate as a function of wavenumber for a weakly baroclinic jet ( $\rho_{\text {shallow }}$ $=27.25 ; S=0.006$ ) under three different bathymetric conditions: a flat bottom, the jet in the retrograde direction, and the jet in the prograde direction. Note that for a flat bottom the growth-rate characteristics for prograde and retrograde jets are identical; hence for flat bottoms only the retrograde version is shown in this and all subsequent figures.

clinic jet, $L_{h}<r_{i}$, indicating the predominance of the barotropic instability. For those runs with the strongly baroclinic jet, it is expected that, because $L_{h} \sim r_{i}$, neither baroclinic or barotropic instabilities will exclusively govern the perturbations' growth. In accord with this expectation, an assessment of the background fields shows that the mean kinetic energy is an order of magnitude larger (smaller) than the mean available potential energy for the weakly (strongly) baroclinic jets.

\section{a. Weakly baroclinic jet}

A stability analysis on a weakly stratified flow ( $\rho_{\text {shallow }}$ $=27.25$ ) was conducted using three different topographies. In all three cases, the structure of the jet remained unchanged; however, either the underlying bathymetry was changed or the jet's flow direction relative to the bathymetry was changed. In one case the jet was retrograde, in another case it was prograde, and in another case the bottom was flat. For all three cases, the jet is unstable over a range of wavenumbers (Fig. 3), with the maximum growth rate occurring between wavenumbers 11 and 13, corresponding to approximately $50 \mathrm{~km}$. (For all of the weakly baroclinic jets, significant growth rates were restricted to the wavenumber range of $0-30$.) Of note is the distinct difference in the magnitude of the growth rates for each of the three cases. For this weakly stratified jet, the retrograde jet is more unstable than that for the flat-bottom case, which is more unstable than the prograde jet. It is apparent that, for weakly stratified jets, topography destabilizes retrograde jets and stabilizes prograde jets.
As seen from Eq. (4.1), downgradient perturbation fluxes are consistent with the growth of the perturbations $\left(\partial \eta^{2} / \partial t>0\right)$ for both the flat-bottom retrograde and prograde jets. With the addition of topography $\left(\partial h_{B} / \partial x<0\right)$, the perturbation flux for the retrograde jet becomes more negative; thus the downgradient flux is increased, resulting in a more unstable jet as evidenced in Fig. 3. For the prograde jet, however, the addition of a topographic slope, where $\left|(\partial z / \partial x)_{\rho}\right|<$ $\left|\partial h_{B} / \partial x\right|$, creates upgradient fluxes, which act to stabilize the flow, as seen in Fig. 3. Calculation of the kinetic and potential energy exchange between the mean and perturbations fields shows that the addition of topography increases the conversion of mean potential energy to eddy potential energy for the retrograde jet, yet for the prograde jet the topographic addition creates a transfer from the eddy potential energy to the mean potential energy, consistent with the upgradient fluxes discussed above. The difference in the conversion between the mean and eddy potential energies is reflected in the growth-rate differences for these three cases.

The difference in the topographic effect on the retrograde and prograde jet is illustrated qualitatively in Fig. 4. Because there is no flow normal to the boundary, the perturbation flow is constrained to lie parallel with the topographic slope. Thus, the degree to which velocity perturbations can create perturbation density fluxes is strongly dependent upon the slope of the isopycnals. For instance, when the isopycnal slope matches the topographic slope, the perturbation density flux will tend to zero because the perturbation velocity is essentially 


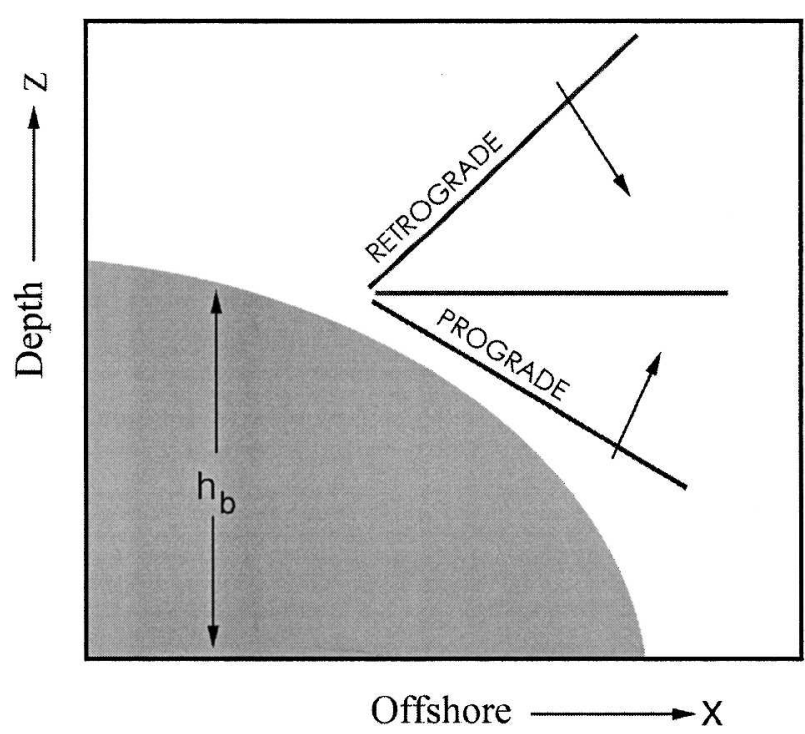

FIG. 4. Schematic showing the effect of a bottom slope and stratification on the orientation of the isopycnals to the bottom.

along a density surface. However, as the "wedge" between the bottom and the isopycnal "opens," the perturbation density fluxes become nonnegligible because the perturbation velocity at the boundary now crosses a density surface. In Fig. 4, the upper (lower) line represents a sloping isopycnal for a retrograde (prograde) jet. When topography is added, it is apparent that downgradient fluxes for the retrograde jet will increase and those for the prograde jet will decrease. The effect of this differing geometry is apparent in Fig. 5 where the perturbation density fluxes (averaged over one wavelength) $\overline{u \rho}$ associated with the fastest-growing wave for the retrograde and prograde jets shown in Fig. 3 are presented. The retrograde jet is characterized by bottom-intensified downgradient fluxes $(\overline{u \rho}<0)$, and the prograde jet is characterized by relatively weak upgradient fluxes (also $\overline{u \rho}<0$ and also bottom-intensified). These fields are consistent with the relationship expressed in Eq. (4.1).

Based on the arguments above, a steepening of the bottom slope is expected to further destabilize a retrograde jet while further stabilizing a prograde jet. To test this expectation, flow characteristics for both retrograde and prograde jets were held constant while the bottom slope was changed. Shown in Fig. 6 are the results of a study in which $\alpha$ was varied to create more and less steep slopes. As evident from Fig. 6a, a steepening of the slope destabilized the retrograde jet; an inspection of Fig. 6b shows the reverse: increasing bottom slopes are associated with decreasing growth rates for prograde jets. It is important to keep in mind that the perturbations are of mixed nature; that is, energy is gained through baroclinic/barotropic instabilities. In both the retrograde and prograde cases, an increasing bottom slope should lead to the stabilization of the
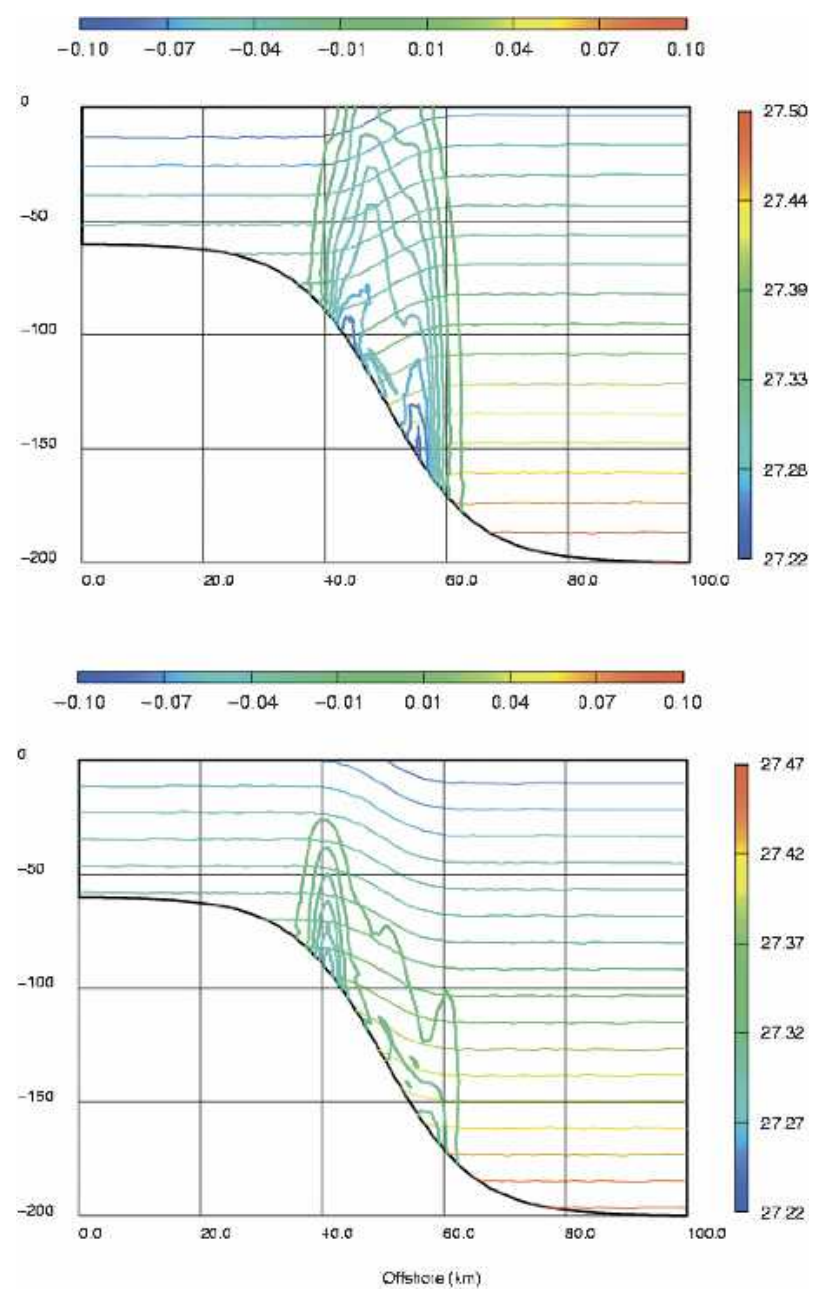

FIG. 5. (top) Cross section of the perturbation density flux $\overline{u \rho}$ for a weakly baroclinic retrograde jet, calculated for the fastestgrowing wave; (bottom) the prograde jet. For both of these jets, $\rho_{\text {shallow }}=27.25$ and $S=0.006$. Depth in meters is given on the vertical axis. The right color bar denotes the scale for the density contours, in sigma- $t$ units; the horizontal bar gives the scale for $\overline{u \rho}$, in dimensionless units. For clarity, flux contours near 0 have been omitted.

barotropic instability. Given that the retrograde jet is destabilized, we surmise that the strong downgradient density fluxes that would increase with increasing slope are sufficient to offset the barotropic tendency for stabilization. Overall, it is important to note that a relatively small change in the bottom slope has a larger impact on the prograde jet than on the retrograde jet, perhaps because the barotropic and baroclinic instabilities both act to stabilize the flow.

Using the geometric argument discussed above, it is readily apparent how a change in the background stratification will affect the stability of these jets. Given that the slope of the isopycnal is determined by

$$
\left(\frac{\partial z}{\partial x}\right)_{\rho}=-\left(\frac{\partial \rho}{\partial x}\right) /\left(\frac{\partial \rho}{\partial z}\right),
$$




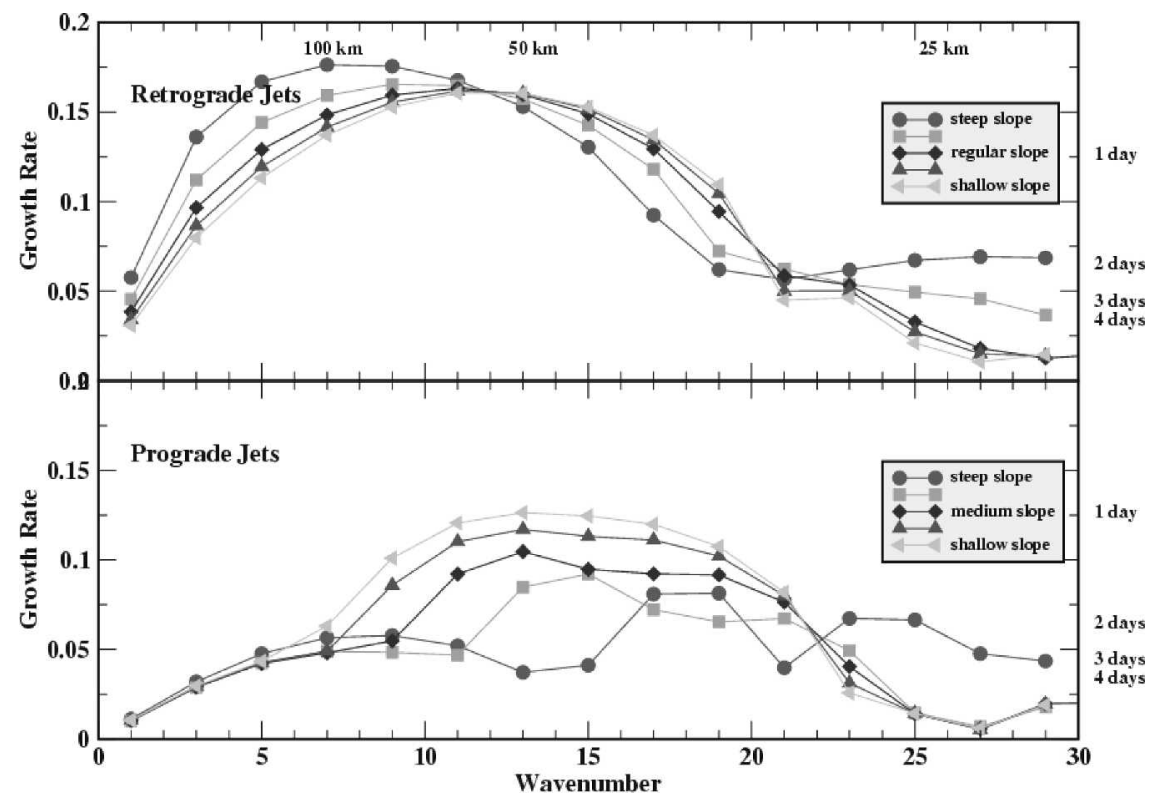

FIG. 6. Growth rates as a function of wavenumber for (top) retrograde and (bottom) prograde jets $\left(\rho_{\text {shallow }}=27.25 ; S=0.006\right)$ with varying bottom slopes. The lateral extent of the bathymetry, $\alpha$ in Eq. (3.14), was varied from $5 \mathrm{~km}$ (more steep) to $25 \mathrm{~km}$ (less steep) in increments of 5 .

an increase in the stratification will act to reduce the magnitude of the isopycnal slope for both the retrograde and prograde jet, as shown in Fig. 4. An increase in stratification essentially flattens the isopycnals for both types of jets, such that a convergence of the stability characteristics at high stratification is expected. To check the validity of this statement, the background stratification was increased for the retrograde and prograde jets used to produce Fig. 3. As seen in Fig. 7a, the retrograde (prograde) jet is indeed stabilized (destabilized) as the stratification is increased. ${ }^{1}$ The growth rates of the two jets converge for strong stratification, when both jets have essentially the same geometric configuration for the isopycnals and bottom slope. This result implies that the barotropic instability for these retrograde and prograde jets is equally affected by the topography, consistent with the Li and McClimans (2000) study of a purely barotropic shelfbreak jet.

\footnotetext{
${ }^{1}$ The prograde 26.0 jet appears to be an exception to the stated hypothesis that increasing stratification destabilizes weakly baroclinic prograde jets. However, such a hypothesis holds only for those modes that are influenced by the bottom, as explained earlier. As shown in LRG, stratification always acts to stabilize a surface-trapped mode. The abrupt change in the prograde 27.25 growth rate curve at $l=9$ (Fig. 7a), along with changes in the phase speed and modal structure (not shown), indicate that the fastest-growing mode has switched from a predominantly barotropic mode to a surface-trapped mode, where the bottom no longer matters. Here then the stratification acts to stabilize the jet and we are left with a 27.25 result that is more stable than the 26.0 jet.
}

Though not shown here, when the highly stratified retrograde and prograde jets are run with a flat bottom, the growth rate increases, reinforcing the result that topography stabilizes a barotropic instability. Of interest is that even though these jets have strong horizontal shear $\left(R_{0}=0.72\right)$, their growth rates are affected by relatively small changes in the orientation of the isopycnals relative to the bottom slope. It is clear that the release of potential energy by the perturbation fluxes at the bottom has a significant effect, though in no cases was the flow completely stabilized by the addition of topography. However, growth rates can vary by more than a factor of 2 on the basis of bottom isopycnal orientation alone (Fig. 7a). Thus, even for weakly baroclinic jets, a small change in the background isopycnal slope can produce growth-rate changes that are effectively distinct ( $<1$ day as compared with 3 days; Fig. $7 a)$. In fact, such changes in the growth rate are comparable in magnitude to those when the horizontal shear of the background flow is doubled (LRG). Thus, relatively small temporal and/or downstream changes in the background structure of the shelfbreak jet could have large consequences for the resultant variability. Of importance is that these changes would depend upon whether the jet was prograde or retrograde.

A calculation of the energy conversions for each of the model runs confirms the expectation that changes in the orientation of the isopycnals relative to the bathymetry affect the energy conversions, in turn affecting the perturbation growth rate. Though the available potential energy is small relative to the mean kinetic energy 


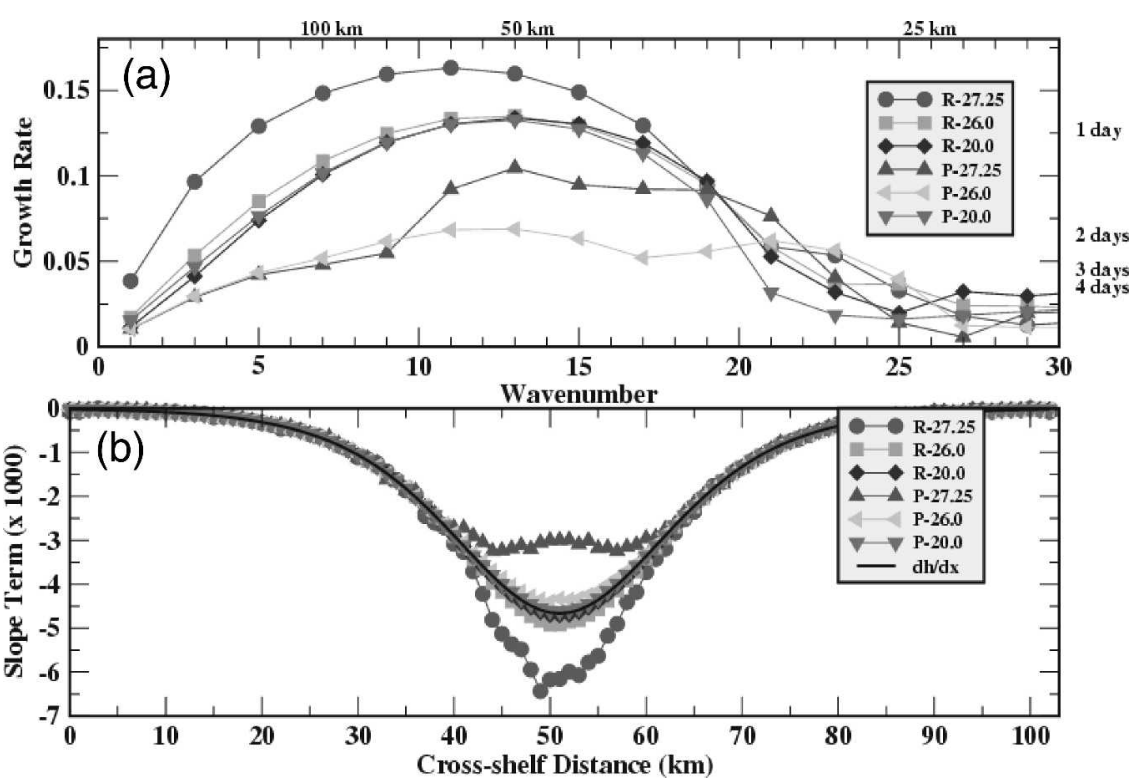

FIG. 7. (a) Growth rates as a function of wavenumber for weakly baroclinic jets with varying stratifications $\left(\rho_{\text {shallow }}=27.25,26.0\right.$, and 20.0 , with $S=0.006,0.033$, and 0.164 , respectively $)$ for both retrograde $(\mathrm{R})$ and prograde $(\mathrm{P})$ jets. (b) The slope term, $-\left[(\partial z / \partial x)_{\rho}-\partial h / \partial x\right]$, corresponding to the jets in (a), calculated at the bottom boundary. The solid black line depicts the cross-shelf bathymetric slope $\partial h_{B} / \partial x$.

for the weakly baroclinic jets, it is not zero, and, as evidenced by the changes in the growth rates when the mean kinetic energy is held constant, its release is significant to the resultant instability. A change in the isopycnal slope relative to the bathymetric slope produces significant changes in the horizontal heat flux term (indicating the transfer of mean potential energy to eddy potential energy). Changes in this energy conversion are reflected in the growth-rate changes.

To test the intuitive understanding of the effect of stratification changes on flow stability, illustrated schematically in Fig. 4 and explained above, the right-hand side of Eq. (4.1) is evaluated as a function of crossstream distance for each of the model jet configurations shown in Fig. 7a. As seen in Fig. 7b, the magnitude of the perturbation density fluxes is maximized near the jet center, primarily because the bottom slope is maximized there, but also because the isopycnal slopes are also maximized there. For the retrograde jet, the downgradient perturbation flux $(\overline{u \rho}<0)$ decreases in magnitude as stratification is increased, thus stabilizing the flow. An increase in stratification for the prograde jet, however, increases the magnitude of the perturbation flux, destabilizing the flow. The convergence, as noted in the growth-rate curves, is also apparent for the perturbation fluxes at strong stratification (Fig. 7b). However, we note that while the increase in stratification for the prograde jet essentially opens the wedge between the bottom slope and the isopycnals, thus creating a larger magnitude for the perturbation density flux, the direction of the fluxes are upgradient.
Because baroclinic instability is characterized by energy exchanges in which the mean available potential energy is lost to the eddy potential energy, which is then transferred to the eddy kinetic energy, it is instructive to examine the structure of the cross-shelf momentum fluxes, $\overline{u v}$, for these unstable jets. Shown in Fig. 8 are the cross-shelf momentum fluxes associated with the fastest-growing wave for some of the weakly and strongly stratified retrograde and prograde jets studied in Fig. 7. The flux fields exhibit a signature of downgradient behavior: the two lobes, split at the $V_{\max }$ location, are of opposite sign, because the mean velocity gradient changes sign at $V_{\max }$. Such a structure implies the importance of the barotropic instability for this high-Rossby-number flow $\left(R_{0}=0.72\right)$. For the weakly stratified jet, the fluxes are nearly uniform in the vertical direction, with a slight surface intensification. The retrograde jet, which has been destabilized by topography, has a relatively stronger flux field than the prograde jet, which was stabilized by topography. Of importance is that the fluxes for these weakly stratified jets are present at the bottom, in contrast to the fluxes for the strongly stratified retrograde and prograde jet. For these jets, the same doubled-lobed structure, centered on $V_{\max }$, is present; however, the flux field is strongly surface intensified such that there is no signature at the bottom. In effect, stratification has insulated the perturbation from the influence of the underlying bathymetry. Such behavior is reminiscent of baroclinic (Eady) modes in which the depth scale of the perturbation is essentially the Rossby height $H_{R}$, given by 
a) Retrograde, Low Stratification

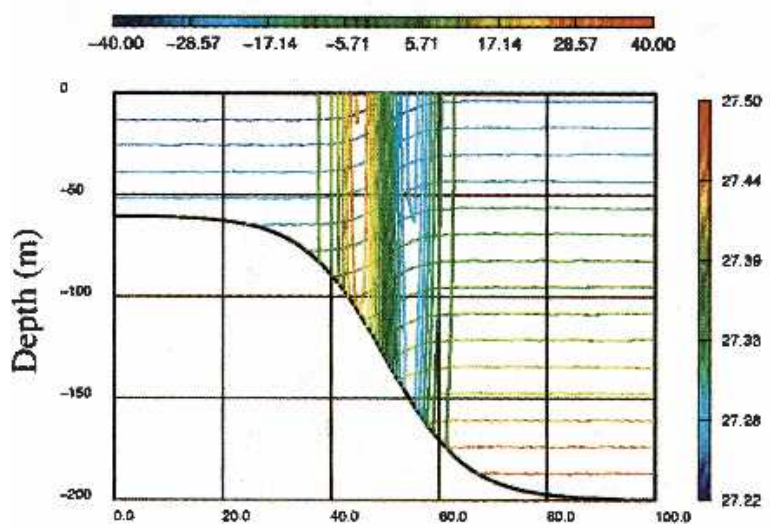

b) Prograde, Low Stratification

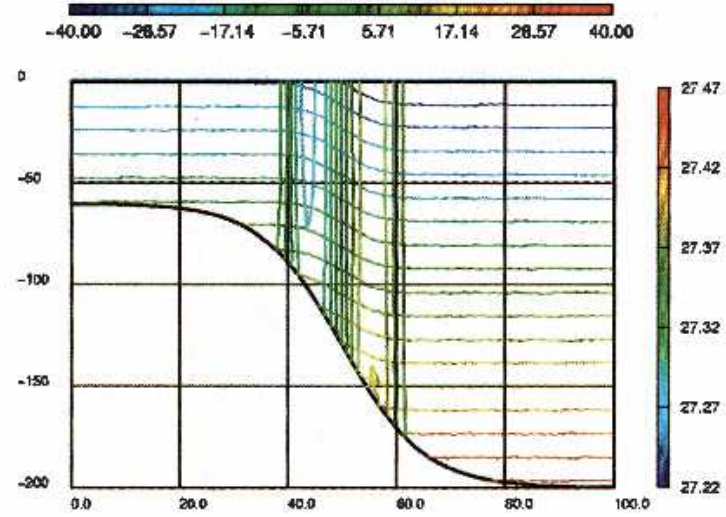

c) Retrograde, High Stratification

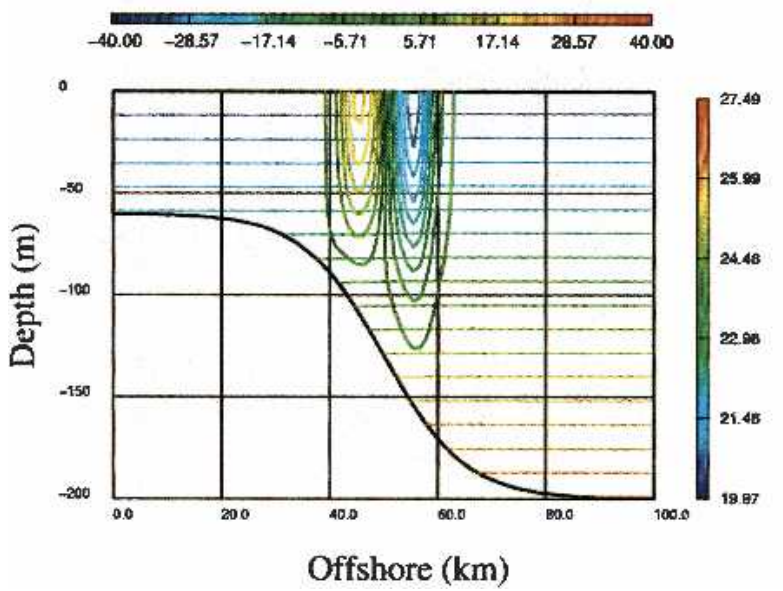

d) Prograde, High Stratification

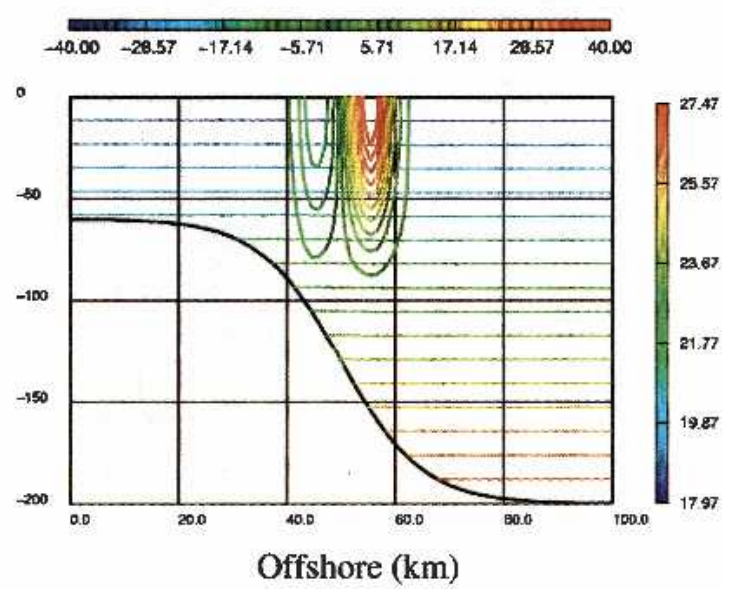

FIG. 8. (a) Cross section of the perturbation momentum flux $\overline{u v}$ for a weakly baroclinic retrograde jet with low background stratification $\left(\rho_{\text {shallow }}=27.25 ; S=0.006\right)$ calculated for the fastest-growing wave. (b) As for (a) but for a prograde jet. (c) Similar to (a) but now for high background stratification $\left(\rho_{\text {shallow }}=20.0 ; S=0.164\right)$. (d) As in (c) but for a prograde jet. Depth in meters is given on the vertical axis.

$f_{0} /\left(N_{0} l\right)($ Gill 1982). As the stratification increases, the Rossby height, where the steering level of the perturbation is located, decreases and the perturbation "moves off" the bottom (Barth 1994). (For the dependence of $H_{R}$ on $l$, the alongfront wavenumber, refer to LRG.) It is noted also that the perturbation momentum flux is greater for the retrograde jet than for the prograde jet, perhaps an indication of the stronger perturbation density flux that results for that jet. Also, the offshore intensification of the perturbation momentum flux for the highly stratified jets suggests the possibility of an asymmetry in cross-frontal exchange.

In summary, topography stabilizes a weakly baroclinic prograde jet and destabilizes a weakly baroclinic retrograde jet. However, as stratification is increased, the prograde jet is destabilized and the retrograde jet is stabilized, resulting in no difference in the growth rates between these jet types. These results essentially show that the geometry of the isopycnal relative to the bottom slope either promotes or stifles perturbation density fluxes that feed the growth of the instability. This result, predicted for quasigeostrophic dynamics, is shown to hold for a range of stratifications and bottom slopes for retrograde and prograde jets governed by primitive equation dynamics. It is interesting that the bottom density fluxes have an impact on the flow stability despite the fact that the flow is strongly barotropic. Different runs in which the background Rossby number varied gave qualitatively the same results; that is, the retrograde/prograde jet dependence on topogra- 


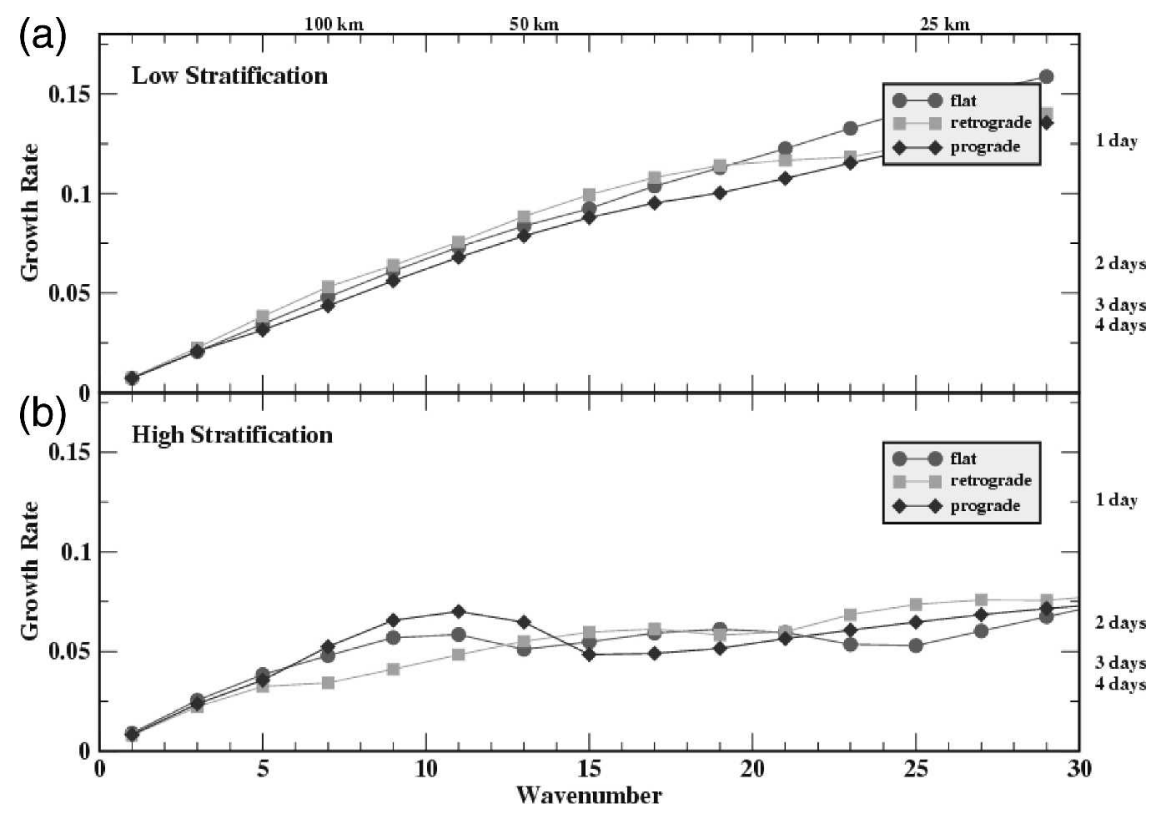

FIG. 9. (a) Plot of growth rate as a function of wavenumber for a strongly baroclinic jet with low stratification $\left(\rho_{\text {shallow }}=27.25\right)$ under three different bathymetric conditions: a flat bottom, a jet in the retrograde direction, and a jet in the prograde direction. (b) As above, but for cases of high stratification $\left(\rho_{\text {shallow }}=20.00\right)$.

phy and stratification was preserved. Also, the effect of the Rossby number on the stability characteristics was unchanged from that reported in LRG; that is, topography was not a modifier of this behavior.

\section{b. Strongly baroclinic jet}

The effect of topography on flow instability was tested for a model jet with considerable baroclinic structure. Unlike the jets studied in the previous section, these baroclinic jets are characterized by strong horizontal and vertical shear. We chose to study the standard jet configuration used in LRG's parameter study: a jet with a depth of $70 \mathrm{~m}, V_{\max }$ of $60 \mathrm{~cm} \mathrm{~s}^{-1}$, and $R_{0}$ of 0.72 , for both weak and strong stratification. As shown in Fig. 9, the growth-rate curves for the flat-bottom case, the retrograde case, and the prograde case exhibit only small differences-particularly so for the weakly stratified jet. As was demonstrated in LRG, the perturbation modes for these baroclinic jets are surface trapped, concentrated where there is appreciable vertical shear (according to their Rossby height, as discussed earlier). Because the perturbation velocities near the sloping boundary would be negligible, it follows that any perturbation density flux would be too weak to affect the overall stability of the jet. It is interesting to note that the effect of increasing stratification for each of these baroclinic jets is to stabilize the flow (see the parameter study conducted in LRG), unlike the cases discussed above in which increasing stratification produced a more (less) stable retrograde (prograde) jet. Because a flattening of the isopycnals generally reduces the potential energy available to feed an instability, reduced growth rates are expected. This expectation, however, is altered when considering the perturbation fluxes near the bottom and their dependence on the orientation of the isopycnal slope relative to the bottom slope, as explained in the previous section.

In addition to being insensitive to the bottom slope, the growth-rate curves for these strongly baroclinic jets differ from those shown in Figs. 3 and 6 in that the growth rate increases with increasing wavenumber and, especially for the low-stratification runs, the maximum growth rate lies beyond the $l=30$ cutoff. As demonstrated by LRG and Barth (1994), the instability of jets with appreciable vertical shear is dominated by a frontal instability mode characterized by smaller wavelengths and smaller vertical scale than the traditional baroclinic (Eady) mode (Moore and Peltier 1989). In all model runs for both the retrograde and the prograde jets, this frontal instability mode was insensitive to the presence of the bottom slope and to changes in the orientation of the background isopycnals to the bottom slope. As noted in section 2, Barth (1994) also noted this insensitivity in his study of coastal upwelling jets. Furthermore, in all parameter studies run in LRG, the frontal instability mode dominated - that is, it had a larger growth rate than the baroclinic mode. Thus, we are left to conclude that for those jets with relatively strong vertical shear, such that the Rossby height $\ll$ water depth, the stability of the jet will be insensitive to the bottom. Our results have shown that the baroclinic mode is sensitive to the bottom configuration, but only 


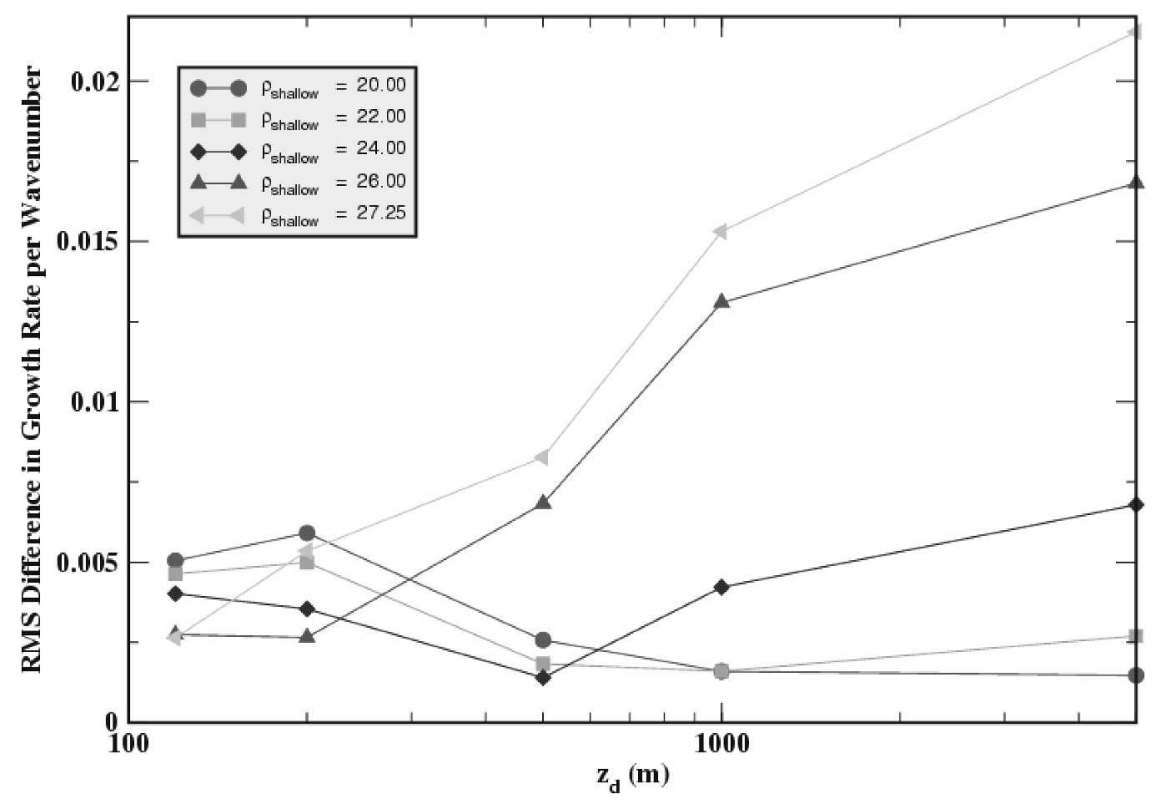

FIG. 10. A semilog plot of the average rms difference in growth rate of the retrograde and prograde jets per wavenumber over the range of wavenumbers $0-20$, illustrating the combined impact of jet depth and background stratification on instability. The horizontal axis is $z_{d}$, as given in Eq. (3.13), and reflects the vertical shear of the jet.

for some jet/slope configurations. Topography has been shown to influence the stability of weakly baroclinic jets, characterized by weak stratification. An increase in the stratification reduces, and eventually eliminates, this dependence. In addition, an increase in the vertical velocity shear such that the jet is more surface intensified reduces and then eliminates the effect of topography on stratification. In both cases, it is the contribution of perturbation density fluxes near the boundary to the instability that has been altered. The degree to which the stratification needs to be increased to eliminate any topographic influence depends strongly upon the strength of the flow field near the bottom boundary. This dependence is shown in Fig. 10 in which the difference in growth rate between a prograde jet and a retrograde jet is plotted as a function of $z_{d}$ (the depth of the $10 \mathrm{~cm} \mathrm{~s}^{-1}$ isotach) for varying background stratifications at the eastern boundary. [Note that for those cases in which $z_{d}$ exceeds the water depth, the implication is that $10 \mathrm{~cm} \mathrm{~s}^{-1}$ would have fallen at $z_{d}$ given the structure of $V(z)$.] For weakly stratified flows $\left(\rho_{\text {shallow }}\right.$ $=27.25$ and 26.0), the effect of topography is seen to increase as $z_{d}$ is increased. In other words, the geometry of the sloping isopycnals at the bottom boundary influences the growth rate for those jets with appreciable velocity shear near the bottom. On the other hand, strongly stratified jets $\left(\rho_{\text {shallow }}=24.0,22.0\right.$, and 20.0) are not affected by the underlying topography over a range of depths. For these cases, the Rossby height is sufficiently removed from the bottom such that the perturbation is insulated from any topographic effects.

\section{Summary}

To ascertain the effect of topography on a shelfbreak frontal current, a linearized primitive equation stability model was employed to determine the three-dimensional propagation of perturbations superposed on a two-dimensional mean flow field, with continuous shear across the current and with depth. The effect of stratification and bottom slope changes was investigated for both retrograde currents and prograde currents. In no instance was a frontal instability mode affected by the presence of the bottom slope, a result attributable to the strong surface trapping of these modes. Their Rossby height is sufficiently small to isolate the perturbation from any bottom effect. Only when the water depth approaches the Rossby height (on the order of 10-20 km) would one expect the bottom topography to influence the stability characteristics of these frontal modes. Because a prior study (LRG) found that these modes generally have faster growth rates than the traditional baroclinic modes, it is expected that for strongly baroclinic shelfbreak flows the topographic influence is negligible. On the other hand, this study has demonstrated that for jets characterized by weak vertical shear (jets referred to in this study as weakly baroclinic) the bottom slope has a strong influence. Although the stabilizing effect of topography on purely barotropic jets is well known, our work has shown that sloping isopycnals associated with weak vertical shear of the jets can influence the growth rate of the instability. We specifically show that the orientation 
of the isopycnal to the bottom slope sets the magnitude of the perturbation density flux, which can exert a stabilizing or destabilizing influence on the flow field. For relatively weak stratification, the presence of a bottom slope destabilizes a retrograde jet and stabilizes a prograde jet. In addition, as the stratification of the retrograde jet is increased, its stability is increased while the stability of the prograde jet is decreased. The change in the growth rate from these stratification changes is as large as the change in growth rate when the Rossby number of the background flow is substantially changed. Thus, one could expect the instability characteristics of a weakly baroclinic jet to change either spatially or temporally as the frontal contrast between the offshore and onshore waters increases or decreases the cross-stream density gradient. Such influence of the bottom on the stability of the flow can be diminished and even negated by an increase in the background stratification. Such an increase effectively decreases the penetration of the perturbation into the water column, essentially isolating the perturbation from any effect of the bottom. Thus, we conclude that only for weakly baroclinic jets with relatively weak stratification will there be a significant influence of the topography on the stability of the flow field.

We note that our study is limited by two simplifying assumptions: symmetric cross-stream shear and uniform stratification at the eastern boundary. The latter assumption is considered to have the most influence on these results. The presence of a strong mixed layer, common in the winter months, could potentially create unstable modes that were not surface trapped. In such cases, it is likely that the topographic influence would be larger than that found here where the fastestgrowing modes were all surface trapped. The incorporation of a mixed layer into this instability model is the planned focus of a future study.

Acknowledgments. The authors thank G. Gawarkiewicz and L. Pratt for their valuable input to this work. MSL gratefully acknowledges support from the Office of Naval Research (N00014-01-1-0260). Computational resources for the study were provided by the North Carolina Supercomputing Center.

\section{REFERENCES}

Barth, J. A., 1994: Shortwave instabilities on coastal jets and fronts. J. Geophys. Res., 99, 16 095-16 115.
Beardsley, R. C., D. C. Chapman, K. H. Brink, S. R. Ramp, and R. Schlitz, 1985: The Nantucket Shoals Flux Experiment (NSFE79). Part I: A basic description of the current and temperature variability. J. Phys. Oceanogr., 15, 713-748.

Blumsack, S. L., and P. J. Gierasch, 1972: Mars: The effects of topography on baroclinic instability. J. Atmos. Sci., 29, 10811089.

Flagg, C. N., and R. C. Beardsley, 1978: On the stability of the shelf water/slope water front south of New England. J. Geophys. Res., 83, 4623-4631.

Fratantoni, P. S., and R. S. Pickart, 2003: Variability of the shelf break jet in the Middle Atlantic Bight: Internally or externally forced? J. Geophys. Res., 108, 3166, doi:10.1029/ 2002JC001326.

Garvine, R. W., K.-C. Wong, G. G. Gawarkiewicz, and R. K. McCarthy, 1988: The morphology of shelfbreak eddies. $J$. Geophys. Res., 93, 15 593-15 607.

Gawarkiewicz, G., 1991: Linear instability models of shelfbreak fronts. J. Phys. Oceanogr., 21, 471-488.

Gill, A. E., 1982: Atmosphere-Ocean Dynamics. Academic Press, $662 \mathrm{pp}$.

Hoskins, B. J., 1975: The geostrophic momentum approximation and semigeostrophic equations. J. Atmos. Sci., 32, 233-242.

Li, S., and T. A. McClimans, 2000: On the stability of barotropic prograde and retrograde jets along a bottom slope. J. Geophys. Res., 105, 8847-8855.

Linder, C., 1996: A climatology of the Middle Atlantic Bight shelfbreak front. M.S. thesis, Dept. of Physical Oceanography, Massachusetts Institute of Technology/Woods Hole Oceanographic Institution, $96 \mathrm{pp}$.

Lozier, M. S., and G. Gawarkiewicz, 2001: Cross-frontal exchange in the Middle Atlantic Bight as evidenced by surface drifters. J. Phys. Oceanogr., 31, 2498-2510.

—, M. S. C. Reed, and G. G. Gawarkiewicz, 2002: Instability of a shelfbreak front. J. Phys. Oceanogr., 32, 924-944.

Mooers, C. N. K., C. N. Flagg, and W. C. Boicourt, 1978: Prograde and retrograde fronts. Oceanic Fronts in Coastal Processes, M. Bowman and W. Esaias, Eds., Springer-Verlag, 43-58.

Moore, G. W. K., and W. R. Peltier, 1987: Cyclogenesis in frontal zones. J. Atmos. Sci., 44, 384-409.

—, and — 1989: Frontal cyclogenesis and the geostrophic momentum approximation. Geophys. Astrophys. Fluid Dyn., 45, 183-197.

Orlanski, I., 1969: The influence of bottom topography on the stability of jets in a baroclinic fluid. J. Atmos. Sci., 26, 12161232.

Pedlosky, J., 1964: The stability of currents in the atmosphere and the ocean: Part I. J. Atmos. Sci., 21, 201-219.

, 1979: Geophysical Fluid Dynamics. Springer-Verlag, 624 pp.

Samelson, R. M., 1993: Linear instability of a mixed-layer front. $J$. Geophys. Res., 98, 10 195-10 204.

Xue, H., and G. Mellor, 1993: Instability of the Gulf Stream front in the South Atlantic Bight. J. Phys. Oceanogr., 23, 23262350. 
Copyright of Journal of Physical Oceanography is the property of American Meteorological Society. The copyright in an individual article may be maintained by the author in certain cases. Content may not be copied or emailed to multiple sites or posted to a listserv without the copyright holder's express written permission. However, users may print, download, or email articles for individual use. 